American Journal of Immunology 8 (1): 1-9, 2012

ISSN 1553-619X

(C) 2012 Science Publications

\title{
Role of IL-10 in Urinary Bladder Carcinoma and Bacillus Calmette-Guerin Immunotherapy
}

\author{
Yi Luo, Eric J. Askeland, Mark R. Newton and Michael A. O’Donnell \\ Department of Urology, University of Iowa, Iowa City, IA 52242, USA
}

\begin{abstract}
Problem statement: Bladder cancer is a common urologic cancer and intravesical Mycobacterium bovis Bacillus Calmette-Guerin (BCG) is the mainstay in the treatment of superficial bladder cancer. However, the current BCG therapy is not desirable with respect to its efficacy and side effects. Interleukin (IL)-10, a T helper type (Th) 2 cytokine, plays an important regulatory role in bladder cancer immunosurveillance and BCG immunotherapy. Therefore, blocking IL-10 activity could be beneficial for bladder cancer patients undergoing BCG therapy. Approach: Treatment with intravesical BCG in combination with systemic IL-10 monoclonal antibody (mAb) specific for IL-10 neutralization or IL-10 receptor (IL-10R) blockage has been evaluated in preclinical bladder cancer models. Results: Addition of anti-IL-10 neutralizing mAb or anti-IL-10R1 mAb enhances BCG induction of Th1 immune responses and anti-bladder cancer immunity. Conclusion/Recommendations: BCG immunotherapy of bladder cancer can be enhanced by addition of IL-10 blocking mAb. Future studies should aim to explore the mechanisms underlying the induction of enhanced antitumor immunity by BCG combination therapy and develop therapeutic regimens for clinical evaluation of the safety and efficacy of BCG combination therapy.
\end{abstract}

Key words: IL-10, bladder cancer, BCG, immunotherapy

\section{INTRODUCTION}

Urinary bladder carcinoma is the second most common urologic neoplasm in the United States, with an estimated 73,510 new cases and 14,880 deaths in 2012 (Siegel et al., 2012). At the time of diagnosis, 20$25 \%$ of cases are muscle invasive (stage T2 or higher) and are typically treated with surgical resection (Williams et al., 2010). The remainders are Nonmuscle Invasive Bladder Cancer (NMIBC) including tumors confined to the epithelial mucosa (Ta), tumors invading the Lamina Propria (T1) and Carcinoma In Situ (CIS). Transurethral Resection of Bladder Tumor (TURBT) is the primary treatment for Ta and T1 lesions. Intravesical therapy is used as adjuvant treatment to prevent recurrence and progression of the disease after TURBT and is also the treatment of choice for CIS. Intravesical instillation of BCG, a widely used vaccine against tuberculosis, is currently the gold standard therapy for NMIBC. BCG therapy results in 50-60\% effectiveness against small residual tumors and a $70-75 \%$ complete response rate for CIS. Unfortunately, a high percentage of patients fail initial BCG therapy and $40-50 \%$ of BCG responders develop recurrent tumors within the first 5 years (Williams et al., 2010). In addition, up to $90 \%$ of patients experience various side effects and rarely lifethreatening complications such as sepsis.

Cytokines are important immunomodulators that can be classified into two major categories, i.e., Th1 cytokines that drive cellular immunity and Th2 cytokines that promote humoral immunity (Fearon and Locksley, 1996). The Th1/Th2 cytokine balance reflects the type of immune responses that occur in the immune system. Studies have revealed a crucial role of Th1 immune response in cancer immunosurveillance including bladder cancer (Mocellin et al., 2001; Brandau and Suttmann, 2007; Sheu et al., 2008). Studies have also shown the dominance of regulatory $\mathrm{T}$ (Treg) cells and Th1 inhibitory cytokines (e.g., TGF- $\beta$ and IL-10) in bladder cancer (Loskog et al., 2007). Consistent with human studies, animal studies have also revealed that Th1 (e.g., IFN- $\gamma$ and IL-12) but not Th2 (e.g., IL-10) cytokines are required for local tumor surveillance (Riemensberger et al., 2002) and that bladder cancer is dominated by IL-10 which inhibit Th1 immune responses at the tumor site (Halak et al., 1999; Yang and Lattime, 2003). It has been known that the therapeutic effect of intravesical BCG is associated with its ability to reverse the disproportion of Th1/Th2

Corresponding Author: Yi Luo, Department of Urology, University of Iowa, 375 Newton Road, 3204 MERF, Iowa City, IA 52242, USA Tel: 319-335-9835 Fax: 319-353-4556 
cytokines, suggesting a crucial role for a Th1 immune response in the intrinsic and immunotherapeutic control of bladder cancer. In this review article we discuss the role of IL-10 in bladder cancer and BCG immunotherapy as well as our progress in BCG combination therapy for bladder cancer. Future direction of study and prospective use of BCG in combination with IL-10 blocking agents are also addressed.

IL-10 and biological activity: IL-10 is a pleiotropic cytokine that functions as a positive or negative mediator in innate and adaptive immunity under different circumstances (Mocellin et al., 2003; 2005). IL-10 is produced by numerous cell types including T cells (Th1, Th2 and Treg) (Fiorentino et al., 1989; Sakaguchi, 2004; O'Garra and Vieira, 2007; Trinchieri, 2007; Maynard et al., 2007), B cells (O'Garra et al., 1990), monocytes/macrophages (Malefyt et al., 1991a), keratinocytes (Enk and Katz, 1992) and epithelial cells (Jung et al., 1995). IL-10 binds to its receptor (IL-10R) expressed on the cell surface, which consists of R1 and R2 subunits and is a class II cytokine receptors (Moore et al., 2001; Ding et al., 2001). T cells, B cells, monocytes/macrophages, Dendritic Cells (DC), Natural Killer (NK) cells, mast cells, granulocytes, keratinocytes and endothelial cells express IL-10R (Moore et al., 2001). The Jak/Stat pathway is involved in IL-10 signaling transduction (Riley et al., 1999). Binding of IL-10 to IL-10R induces Jak1 and Tyk2, resulting in Stat1, Stat3 and Stat5 activation, dimerization and unclear translocation and subsequent transcription of target genes that leads to IL-10 mediated biological activities (Finbloom and Winestock, 1995; Wehinger et al., 1996).

IL-10 has been shown to inhibit cellular immune responses via a number of mechanisms. IL-10 can block the accumulation of macrophages and DC at the tumor site (Richter et al., 1993; Qin et al., 1997) and down-regulate the expression of MHC class II and costimulatory molecules (e.g., B7 and ICAM-1) on these cells (Malefyt et al., 1991b; Ding et al., 1993; Willems et al., 1994; O'Garra and Murphy, 2009), thus suppressing the induction of specific immune responses. IL-10 can also reduce DC's capacity to stimulate $\mathrm{T}$ cells, leading to the induction of antigenspecific anergy of $\mathrm{T}$ cells (Groux et al., 1996; Steinbrink et al., 1997; 2002; Zeller et al., 1999). It has also been reported that CD4+ T cells in the presence of IL-10 during activation can differentiate into $T$ regulatory cells 1 (Tr1) that are responsible for peripheral immune tolerance induced by IL-10 (Groux et al., 1997). In addition, IL-10 can also prevent release of cytokines (e.g., IFN- $\gamma$ and TNF- $\alpha$ ) and reactive nitrogen/oxygen intermediates (e.g., NO) by macrophages and NK cells (Bogdan et al., 1991; Fiorentino et al., 1991; Cenci et al., 1993), thus inhibiting inflammatory and tumoricidal activities of these cells.

Although IL-10 was initially named a cytokine synthesis inhibitory factor and is classified as a Th2 cytokine (Moore et al., 2001), recent studies revealed that Th1 cells have an ability to produce IL-10 for the feedback control of their own activities (O'Garra and Vieira, 2007; Trinchieri, 2007). In addition to suppressive effects, IL-10 also exerts some stimulatory effects, favoring immune-mediated rejection of cancer that has been evidenced in a large body of animal studies (Zheng et al., 1996; Berman et al., 1996; Kundu et al., 1996; Kundu and Fulton, 1997; Fujii et al., 2001; Segal et al., 2002; Tanikawa et al., 2012). IL-10 can promote Cytotoxic T Lymphocyte (CTL) differentiation and expansion (MacNeil et al., 1990; Chen and Zlotnik, 1991; Groux et al., 1998; Rowbottom et al., 1999; Fujii et al., 2001), enhance NK cell cytolytic activity (Zheng et al., 1996; Kundu et al., 1996; Kundu and Fulton, 1997), induce DC antigen uptake (Morel et al., 1997; Allavena et al., 1998; Fortsch et al., 2000), increase leukocyte IFN- $\gamma$ production (Tilg et al., 2002) and inhibit Treg cell development (Tanikawa et al., 2012). In addition, IL-10 can also inhibit cancer development and progression through its regulatory effects on inflammatory cytokine production, as inflammation is often associated with increased tumor angiogenesis and invasiveness (Mocellin et al., 2003). Thus, IL-10 plays a dual role in tumor-associated immune responses, either promoting antitumor immune responses or mediating tumor escape from immune surveillance. The biological activities of IL-10 appear to be context dependent and vary in different cancer model systems.

IL-10 in bladder cancer: IL-10 functions as a Th1 inhibitory cytokine in bladder cancer. Studies have revealed that patients with bladder cancer develop a Th2 dominant status with a deficient Th1 immune response. Increased levels of IL-10 and other Th2 cytokines (e.g., IL-4, IL-5 and IL-6), along with decreased levels of Th1 cytokines (e.g., IFN- $\gamma$ and IL2 ), have been observed in the serum of bladder cancer patients (Agarwal et al., 2010; Satyam et al., 2011). This circulating cytokine profile seems to correlate well with the grade and severity of bladder cancer. Peripheral CD4+ $\mathrm{T}$ cells from bladder cancer patients have also been observed to express increased IL-10 and other Th2 cytokines, along with decreased Th1 cytokines (Agarwal et al., 2006). Consistent with the systemic cytokine profiles, increased concentrations of 
IL-10 and other Th2 cytokines (e.g., IL-13) have been observed in the urine of bladder cancer patients (Margel et al., 2011). A recent study suggested that urinary IL6/IL-10 ratio might be useful as a prognostic marker of recurrence in patients with intermediate risk superficial bladder cancer (Cai et al., 2007). To support this local Th2 dominant status, IL-10 has been detected in bladder cancer specimens at both protein and mRNA levels (Cardillo et al., 2000; Loskog et al., 2007).

All animal studies consistently support the Th2 cytokine dominance observed in human bladder cancer. Studies have demonstrated that IFN- $\gamma$ and IL-12 but not IL-10 are required for local tumor surveillance in a syngeneic mouse model of bladder cancer (Riemensberger et al., 2002). Mice genetically deficient in IFN- $\gamma$ (IFN- $\gamma-/-)$ or IL-12 (IL-2-/-) died much earlier than wild-type mice after tumor implantation; however, this intrinsic antitumor response was not altered in IL$10^{-/-}$mice. Studies have also shown that a marked polarization exists towards the expression of $\mathrm{Th} 2$ cytokines, including IL-10, during progression of bladder cancer in a syngeneic mouse model (Tham et al., 2011). Studies have also revealed that MB49 cells, a widely-used mouse bladder cancer cell line (Chen et al., 2009), can induce IL-10 production by infiltrating immune cells, resulting in inhibition of specific antitumor immune responses (Halak et al., 1999). This inhibition has been attributed to DC dysfunction for $\mathrm{T}$ cell stimulation resulting from tumor-induced IL-10 production (Yang and Lattime, 2003). Studies have also revealed the ability of macrophages to inhibit bladder cancer cell growth in vitro; however, this macrophage mediated growth arrest can be reversed by the addition of IL-10 (Dufresne et al., 2011), suggesting that IL-10 is inhibitory to macrophage tumoricidal activity in vivo. All these findings suggest that therapeutic strategies for Th1 induction and Th2 dampening may be effective in the treatment of bladder cancer.

There are limited studies on IL-10 gene polymorphisms in bladder cancer. IL-10 functions in a highly complex and coordinated manner and its gene variations may lead to altered production and activity of this cytokine, thus affecting the susceptibility to bladder cancer. A recent study revealed that two single nucleotide polymorphisms of IL-10 gene at G-1082A and C-819T positions are associated with increased bladder cancer risk and may be useful as a molecular marker of bladder cancer (Ahirwar et al., 2009). Similarly, another study showed that IL-10 gene polymorphisms (-1082GG and $-1082 \mathrm{GCC} / \mathrm{GCC}$ ) are potential risk factors for bladder cancer progression and may even alter the effect of BCG immunotherapy
(Basturk et al., 2006). These observations warrant further investigation on genetic markers for bladder cancer diagnosis, prognosis and treatment.

IL-10 in BCG treatment of bladder cancer: The effect of BCG immunotherapy on bladder cancer depends on the proper induction of Th1 immune responses. Intravesical instillation of BCG induces a complex inflammatory cascade in the bladder mucosa, reflecting activation of multiple types of immune cells and bladder tissue cells (Ratliff, 1991; Brandau and Suttmann, 2007; Alexandroff et al., 2010). Following BCG instillation, various leukocyte types infiltrate into the bladder wall including neutrophils, monocytes/macrophages, lymphocytes, NK cells and DC (Bohle et al., 1990a; Prescott et al., 1992; Saban et al., 2007). These infiltrating leukocytes, together with activated urothelial cells, produce numerous proinflammatory cytokines and chemokines. Subsequently, a transient massive amount of cytokines and chemokines (Bohle et al., 1990b; Boer et al., 1991a; De Reijke et al., 1996; Taniguchi et al., 1999; Saint et al., 2002; Nadler et al., 2003; Luo et al., 2007), together with a variety of leukocyte types (Boer et al., 1991a-c; Simons et al., 2008), can be detected in voided urine. The urine of mice treated with intravesical BCG also exhibits increased concentrations of cytokines and chemokines (Saban et al., 2007). In addition to local immune induction, intravesical BCG also results in systemic immune responses as manifested by increased levels of cytokines and chemokines in the serum (Elsasser-Beile et al., 2000; Magno et al., 2002).

It has been noted that the development of a dominant Th1 cytokine profile (e.g., IFN- $\gamma$, IL-2 and IL-12) is associated with the therapeutic effect of BCG, whereas the presence of a high level of Th2 cytokines (e.g., IL-10) is associated with BCG failure (De Reijke et al., 1996; Saint et al., 2002; Nadler et al., 2003). The kinetic analysis of urinary cytokines indicates that the production of IFN- $\gamma$, IL-2 and IL-12 precedes the production of IL-10 in patients undergoing BCG therapy (Nadler et al., 2003). A tendency toward higher ratios of IFN- $\gamma$ vs. IL-10 has been observed for BCG responders (Saint et al., 2001; 2002; Nadler et al., 2003). IL-10, as a major Th2 cytokine, appears to play an important inhibitory role in BCG immunotherapeutic control of bladder cancer. To support this, it has been reported that intravesical BCG induces markedly increased local immune responses in $\mathrm{IL}-10^{-/-}$mice, coinciding with increased therapeutic efficacy compared to wild-type mice (Riemensberger et al., 2002). In line with this, we have observed that both IL$10^{-/-}$mice and mice treated with anti-IL-10 neutralizing 
mAb develop enhanced BCG induced anti-bladder cancer immunity in a syngeneic mouse orthotopic tumor model (Nadler et al., 2003). We have also demonstrated that blocking IL-10 signal transduction by anti-IL-10R1 mAb that binds to IL-10R1 enhances BCG-induced anti-bladder cancer immunity in a similar mouse model of bladder cancer (Bockholt et al., 2012). In addition to in vivo studies, we have observed the inhibitory effect of IL-10 on BCG induced macrophage cytotoxicity against bladder cancer cells in vitro through antibody neutralization of IL-10 for wild-type macrophage cultures or use of IL-10 ${ }^{-/-}$macrophages in assays (Luo et al., 2010).

Blocking IL-10 enhances BCG-induced Th1 immune responses and anti-bladder cancer immunity: Bladder cancer is dominated by a Th2 polarized immunopathologic response. Intravesical BCG therapy can shift the Th2 environment toward a Th1 milieu, a phenomenon linked to the therapeutic effect of BCG on treating bladder cancer. Although BCG therapy has long been proven to be effective for treating bladder cancer, 30-40\% of patients fail BCG therapy and 40$50 \%$ of BCG responders develop recurrent tumors (Williams et al., 2010). Therefore, the current BCG therapy is not desirable with respect to its efficacy. Since evidence supports the Th1 immune response to be essential in BCG-mediated bladder cancer destruction, studies have focused on enhancing the BCG induction of Th1 immune responses. During the past 2 decades, we and others have developed a strategy to combine BCG with Th1-stimulating cytokines (e.g., IFN- $\alpha$ ) to enhance BCG induction of Th1 immune responses (Luo et al., 1999; O'Donnell et al., 1999; O'Donnell and Boehle, 2006). In addition, we have also combined BCG with IL-10 blocking agents, either at the protein or receptor level, to enhance BCG induction of Th1 immune responses. We have observed that blocking IL10 enhances BCG-induced Th1 immune responses and anti-bladder cancer immunity (Nadler et al., 2003; Bockholt et al., 2012).

Our early studies showed that absence of IL-10 abrogated either by systemic anti-IL-10 neutralizing $\mathrm{mAb}$ or the use of IL-10 $0^{-/-}$mice resulted in enhanced delayed-type hypersensitivity (DTH) responses that were associated with increased mononuclear cell infiltration and Th1 cytokine production (e.g., IFN- $\gamma$ ) in the BCG-treated bladders (Nadler et al., 2003). Under the condition of enhanced DTH responses, a significant enhancement in BCG-induced anti-bladder cancer immunity was observed in a syngeneic mouse orthotopic tumor model (Nadler et al., 2003), suggesting that blocking IL-10 production and/or activity may have therapeutic values for BCG immunotherapy of bladder cancer.

In addition to IL-10 neutralization, we recently evaluated the effect of IL-10 blockage at the receptor level on BCG induction of Th1 immune responses and anti-bladder cancer immunity (Bockholt et al., 2012). Mice treated with intravesical BCG plus systemic antiIL-10R1 mAb showed significantly increased IFN- $\gamma$ mRNA and protein in the bladder and urine, respectively, in a dose-dependent manner. Accordingly, under the bladder cancer cell implantation condition, mice treated with BCG in combination with anti-IL10R1 mAb showed substantially improved tumor-free and survival rates compared to control mice. Studies further revealed that the combination therapy with a reduced dose (1/3 full-dose) of BCG could significantly prevent bladder cancer metastasis to the lung during an extended experimental period (no metastasis in mice treated with combination therapy vs. 36-53\% of incidence in control mice) (our unpublished observations). These observations suggest that anti-IL10R1 mAb could serve as an effective agent for treating bladder cancer, particularly for high-risk patients, when combined with BCG.

\section{CONCLUSION}

Bladder cancer is a common urologic malignancy dominated by a Th2 polarized immunopathologic response. Intravesical BCG can shift the Th2 environment toward a Th1 milieu, leading to effective anti-bladder cancer immunity in the majority of patients. However, the current BCG therapy is associated with high disease recurrence and progression as well as a lack of therapeutic response in some patients. In addition, BCG-associated side effects are common and occasionally life-threatening. The efficacy of BCG can be improved by combination with Th1stimulating agents, such as Th1 cytokines and Th2 blocking agents. Since IL-10 plays an important regulatory role in bladder cancer immunosurveillance and BCG immunotherapy, blocking IL-10 activity could enhance BCG induction of Th1 immunity and therapeutic control of bladder cancer. This assumption has been proven to be true in our animal studies. We combined BCG with anti-IL-10 neutralizing mAb or anti-IL-10R1 $\mathrm{mAb}$ to treat bladder cancer in a syngeneic mouse orthotopic tumor model and found that these combination therapies were more effective than BCG monotherapy. We further observed that a $1 / 3$ full-dose of BCG in combination with anti-IL-10R1 $\mathrm{mAb}$ effectively prevented bladder cancer metastasis to the lung. These observations suggest that BCG could be 
used at a reduced dose when combined with an IL-10 blocking agent to minimize BCG side effects while maintaining BCG efficacy. Future studies should aim to investigate the mechanisms through which BCG combination therapy induces enhanced anti-bladder cancer immunity and develop therapeutic regimens to evaluate the safety and efficacy of BCG combination therapy in clinical settings.

\section{ACKNOWLEDGEMENT}

We would like to thank Ms. Kris Greiner for editorial review of this article.

\section{REFERENCES}

Agarwal, A., S. Verma, U. Burra, N.S. Murthy and N.K. Mohanty et al., 2006. Flow cytometric analysis of Th1 and Th2 cytokines in PBMCs as a parameter of immunological dysfunction in patients of superficial transitional cell carcinoma of bladder. Cancer Immunol. Immunother., 55: 734743. PMID: 16283306

Agarwal, A., U. Agrawal, S. Verma, N.K. Mohanty and S. Saxena, 2010. Serum Th1 and Th2 cytokine balance in patients of superficial transitional cell carcinoma of bladder pre- and post-intravesical combination immunotherapy. Immunopharmacol. Immunotoxicol., 32: 348-356. PMID: 20105083

Ahirwar, D., A. Mandhani and R.D. Mittal, 2009. Interleukin-10 G-1082A and C-819T polymorphisms as possible molecular markers of urothelial bladder cancer. Arch. Med. Res., 40: $97-$ 102. PMID: 19237018

Alexandroff, A.B., S. Nicholson, P.M. Patel and A.M. Jackson, 2010. Recent advances in bacillus Calmette-Guerin immunotherapy in bladder cancer. Immunother., 2: 551-560. PMID: 20636008

Allavena, P., L. Piemonti, D. Longoni, S. Bernasconi and A. Stoppacciaro et al., 1998. IL-10 prevents the differentiation of monocytes to dendritic cells but promotes their maturation to macrophages. Eur. J. Immunol., 28: 359-69. PMID: 9485215

Basturk, B., I. Yavascaoglu, B. Oral, G. Göral and B. Oktay, 2006. Cytokine gene polymorphisms can alter the effect of Bacillus Calmette-Guérin (BCG) immunotherapy. Cytokine, 35: 1-5. PMID: 16938461

Berman, R.M., T. Suzuki, H. Tahara, P.D. Robbins and S.K. Narula et al., 1996. Systemic administration of cellular IL-10 induces an effective, specific and long-lived immune response against established tumors in mice. J. Immunol., 157: 231-238. PMID: 8683120
Bockholt, N.A., M.J. Knudson, J.R. Henning, J.L. Maymi and P. Weady et al., 2012. Anti-IL-10R1 monoclonal antibody enhances BCG-induced TH1 immune responses and antitumor immunity in a mouse orthotopic model of bladder cancer. J. Urol.

Bogdan, C., Y. Vodovotz and C. Nathan, 1991. Macrophage deactivation by interleukin 10. J. Exp. Med., 174: 1549-1555. PMID: 1744584

Bohle, A., C. Nowc, A.J. Ulmer, J. Musehold and J. Gerdes et al., 1990a. Elevations of cytokines interleukin-1, interleukin-2 and tumor necrosis factor in the urine of patients after intravesical bacillus Calmette-Guerin immunotherapy. J. Urol., 144: 59-64. PMID: 2193171

Bohle, A., J. Gerdes, A.J. Ulmer, A.G. Hofstetter and H.D. Flad, 1990b. Effects of local bacillus Calmette-Guerin therapy in patients with bladder carcinoma on immunocompetent cells of the bladder wall. J. Urol., 144: 53-58. PMID: 2359181

Brandau, S. and H. Suttmann, 2007. Thirty years of BCG immunotherapy for non-muscle invasive bladder cancer: A success story with room for improvement. Biomed. Pharmacother., 61: 299305. PMID: 17604943

Boer, E.C.D., W.H. De Jong, P.A. Steerenberg, A.P. Van Der Meijden and L.A. Aarden et al., 1991a. Leukocytes and cytokines in the urine of superficial bladder cancer patients after intravesical immunotherapy with bacillus Calmette-Guerin. In Vivo, 5: 671-677. PMID: 1810454.

Boer, E.C.D., W.H. De Jong, A.P. Van Der Meijden, P.A. Steerenberg and F. Witjes et al., 1991b. Leukocytes in the urine after intravesical BCG treatment for superficial bladder cancer. A flow cytofluorometric analysis. Urol. Res., 19: 45-50. PMID: 2028562.

Boer, E.C.D., W.H. De Jong, A.P. Van Der Meijden, P.A. Steerenberg and J.A. Witjes et al., 1991c. Presence of activated lymphocytes in the urine of patients with superficial bladder cancer after intravesical immunotherapy with bacillus Calmette-Guérin. Cancer Immunol. Immunother., 33: 411-416. PMID: 1878894.

Boer, T.M.D., E.C. De Boer, K.H. Kurth and D.H. Schamhart, 1996. Urinary cytokines during intravesical bacillus Calmette-Guerin therapy for superficial bladder cancer: Processing, stability and prognostic value. J. Urol., 155: 477-482. PMID: 8558640

Cai, T., S. Mazzoli, F. Meacci, G. Tinacci and G. Nesi et al., 2007. Interleukin-6/10 ratio as a prognostic marker of recurrence in patients with intermediate risk urothelial bladder carcinoma. J. Urol., 178: 1906-1911. PMID: 17868727 
Cardillo, M.R., P. Sale and F. Di Silverio, 2000. Heat shock protein-90, IL-6 and IL-10 in bladder cancer. Anticancer Res., 20: 4579-4583. PMID: 11205307

Cenci, E., L. Romani, A. Mencacci, R. Spaccapelo and E. Schiaffella et al., 1993. Interleukin-4 and interleukin-10 inhibit nitric oxide-dependent macrophage killing of Candida albicans. Eur. J. Immunol., 23: 1034-1038. PMID: 8477799

Chen, F., G. Zhang, Y. Cao, M.J. Hessner and W.A. See, 2009. MB49 murine urothelial carcinoma: molecular and phenotypic comparison to human cell lines as a model of the direct tumor response to bacillus Calmette-Guerin. J. Urol., 182: 2932-2937. PMID: 19853870

Chen, W.F. and A. Zlotnik, 1991. IL-10: A novel cytotoxic $\mathrm{T}$ cell differentiation factor. J. Immunol., 147: 528-534. PMID: 1906502

Ding, L., P.S. Linsley, L.Y. Huang, R.N. Germain and E.M. Shevach, 1993. IL-10 inhibits macrophage costimulatory activity by selectively inhibiting the up-regulation of B7 expression. J. Immunol., 151: 1224-1234. PMID: 7687627

Ding, Y., L. Qin, D. Zamarin, S.V. Kotenko and S. Pestka, et al., 2001. Differential IL-10R1 expression plays a critical role in IL-10-mediated immune regulation. J. Immunol., 167: 6884-6892. PMID: 11739506

Dufresne, M., G. Dumas, E. Asselin, C. Carrier and M. Pouliot et al., 2011. Pro-inflammatory type-1 and anti-inflammatory type-2 macrophages differentially modulate cell survival and invasion of human bladder carcinoma T24 cells. Mol. Immunol., 48: 1556-1567. PMID: 21601924

Elsasser-Beile, U., O. Gutzeit, S. Bauer, A. Katzenwadel and W. Schultze-Seemann et al., 2000. Systemic and local immunomodulatory effects of intravesical BCG therapy in patients with superficial urinary bladder carcinomas. J. Urol., 163: 296-299. PMID: 10604379.

Enk, A.H. and S.I. Katz, 1992. Identification and induction of keratinocyte-derived IL-10. J. Immunol., 149: 92-95. PMID: 1607665.

Fearon, D.T. and R.M. Locksley, 1996. The instructive role of innate immunity in the acquired immune response. Science, 272: 50-53. PMID: 8600536.

Finbloom, D.S. and K.D. Winestock, 1995. IL-10 induces the tyrosine phosphorylation of tyk 2 and Jak1 and the differential assembly of STAT1 alpha and STAT3 complexes in human $\mathrm{T}$ cells and monocytes. J. Immunol., 155: 1079-1090. PMID: 7543512
Fiorentino, D.F., A. Zlotnik, T.R. Mosmann, M. Howard and A. O'Garra, 1991. IL-10 inhibits cytokine production by activated macrophages. J. Immunol., 147: 3815-3822. PMID: 1940369

Fiorentino, D.F., M.W. Bond and T.R. Mosmann, 1989. Two types of mouse $\mathrm{T}$ helper cell. IV. Th2 clones secrete a factor that inhibits cytokine production by Th1 clones. J. Exp. Med., 170: 2081-2095. PMID: 2531194

Fortsch, D., M. Rollinghoff and S. Stenger, 2000. IL-10 converts human dendritic cells into macrophagelike cells with increased antibacterial activity against virulent Mycobacterium tuberculosis. J. Immunol., 165: 978-987. PMID: 10878374.

Fujii, S., K. Shimizu, T. Shimizu and M.T. Lotze, 2001. Interleukin-10 promotes the maintenance of antitumor CD8 (+) T-cell effector function in situ. Blood, 98: 2143-2151. PMID: 11568001

Groux, H., A. O'Garra, M. Bigler, M. Rouleau and S. Antonenko et al., 1997. A CD4+ T-cell subset inhibits antigen-specific T-cell responses and prevents colitis. Nature, 389: 737-742. PMID: 9338786

Groux, H., M. Bigler, J.E. De Vries and M.G. Roncarolo, 1996. Interleukin-10 induces a longterm antigen-specific anergic state in human CD4+ T cells. J. Exp. Med., 184: 19-29. PMID: 8691133.

Groux, H., M. Bigler, J.E. De Vries and M.G. Roncarolo, 1998. Inhibitory and stimulatory effects of IL-10 on human CD8+ T cells. J. Immunol., 160: 3188-3193. PMID: 9531274

Halak, B.K., H.C. Maguire Jr and E.C. Lattime, 1999. Tumor-induced interleukin-10 inhibits type 1 immune responses directed at a tumor antigen as well as a non-tumor antigen present at the tumor site. Cancer Res., 59: 911-917. PMID: 10029084.

Jung, H.C., L. Eckmann, S.K. Yang, A. Panja and J. Fierer et al., 1995. A distinct array of proinflammatory cytokines is expressed in human colon epithelial cells in response to bacterial invasion. J. Clin. Invest., 95: 55-65. PMID: 7814646

Kundu, N., T.L. Beaty, M.J. Jackson and A.M. Fulton, 1996. Antimetastatic and antitumor activities of interleukin 10 in a murine model of breast cancer. J. Natl. Cancer Inst., 88: 536-541. PMID: 8606382

Kundu, N. and A.M. Fulton, 1997. Interleukin-10 inhibits tumor metastasis, downregulates $\mathrm{MHC}$ class I and enhances NK lysis. Cell. Immunol., 180: 55-61. PMID: 9316639

Loskog, A., C. Ninalga, G. Paul-Wetterberg, M. De La Torre and P.U. Malmström et al., 2007. Human bladder carcinoma is dominated by T-regulatory cells and Th1 inhibitory cytokines. J. Urol., 177: 353-358. PMID: 17162090 
Luo, Y., Chen, X., T.M. Downs, W.C. DeWolf and M.A. O'Donnell, 1999. IFN-alpha 2B enhances Th1 cytokine responses in bladder cancer patients receiving Mycobacterium bovis bacillus CalmetteGuérin immunotherapy. J. Immunol., 162: 23992405. PMID: 9973521

Luo, Y., X. Chen and M.A. O'Donnell, 2007. Mycobacterium bovis bacillus Calmette-Guérin (BCG) induces human CC- and CXC-chemokines in vitro and in vivo. Clin. Exp. Immunol., 147: 370-378. PMID: 17223980

Luo, Y., R. Han, D.P. Evanoff and X. Chen, 2010. Interleukin-10 inhibits Mycobacterium bovis bacillus Calmette-Guerin (BCG)-induced macrophage cytotoxicity against bladder cancer cells. Clin. Exp. Immunol., 160: 359-368. PMID: 20148913

Malefyt, R.D.W., J. Abrams, B. Bennett, C.G. Figdor and J.E. De Vries, 1991a. Interleukin 10(IL-10) inhibits cytokine synthesis by human monocytes: an autoregulatory role of IL-10 produced by monocytes. J. Exp. Med., 174: 1209-1220. PMID: 1940799

Malefyt, R.D.W., J. Haanen, H. Spits, M.G. Roncarolo and A. Te Velde et al., 1991b. Interleukin 10 (IL10) and viral IL-10 strongly reduce antigenspecific human $\mathrm{T}$ cell proliferation by diminishing the antigen-presenting capacity of monocytes via downregulation of class II major histocompatibility complex expression. J. Exp. Med., 174: 915-924. PMID: 1655948

MacNeil, I.A., T. Suda, K.W. Moore, T.R. Mosmann and A. Zlotnik, 1990. IL-10, a novel growth cofactor for mature and immature $T$ cells. $\mathrm{J}$. Immunol., 145: 4167-4173. PMID: 2124236

Magno, C., D. Melloni, A. Galì, G. Mucciardi and G. Nicocia et al., 2002. The anti-tumor activity of bacillus Calmette-Guerin in bladder cancer is associated with an increase in the circulating level of interleukin-2. Immunol. Lett., 81: 235-238. PMID: 11947931

Margel, D., M. Pevsner-Fischer, J. Baniel, O. Yossepowitch and I.R. Cohen, 2011. Stress proteins and cytokines are urinary biomarkers for diagnosis and staging of bladder cancer. Eur. Urol., 59: 113-119. PMID: 20970244

Maynard, C.L., L.E. Harrington, K.M. Janowski, J.R. Oliver and C.L. Zindl et al., 2007. Regulatory T cells expressing interleukin 10 develop from Foxp3+ and Foxp3- precursor cells in the absence of interleukin 10. Nat. Immunol., 8: 931-941. PMID: 17694059
Mocellin, S., E. Wang and F.M. Marincola, 2001. Cytokines and immune response in the tumor microenvironment. J. Immunother., 24: 392-407. PMID: 11696695

Mocellin, S., M.C. Panelli, E. Wang, D. Nagorsen and F.M. Marincola, 2003. The dual role of IL-10. Trends Immunol., 24: 36-43. PMID: 12495723

Mocellin, S., F.M. Marincola and H.A. Young, 2005. Interleukin-10 and the immune response against cancer: A counterpoint. J. Leukoc. Biol., 78: 104351. PMID: 16204623

Moore, K.W., R. De Waal Malefyt, R.L. Coffman and A. O'Garra, 2001. Interleukin-10 and the interleukin-10 receptor. Annu. Rev. Immunol., 19: 683-765. PMID: 11244051

Morel, A.S., S. Quaratino, D.C. Douek and M. Londei, 1997. Split activity of interleukin-10 on antigen capture and antigen presentation by human dendritic cells: Definition of a maturative step. Eur. J. Immunol., 27: 26-34. PMID: 9021994

Nadler, R., Y. Luo, W. Zhao, J.K. Ritchey and J.C. Austin, et al., 2003. Interleukin 10 induced augmentation of Delayed-Type Hypersensitivity (DTH) enhances Mycobacterium bovis bacillus Calmette-Guérin (BCG) mediated antitumour activity. Clin. Exp. Immunol., 131: 206-216. PMID: 12562379

O'Donnell, M.A., Y. Luo, X. Chen, A. Szilvasi and S.E. Hunter, et al., 1999. Role of IL-12 in the induction and potentiation of IFN-gamma in response to bacillus Calmette-Guérin. J. Immunol., 163: 42464252. PMID: 10510362

O'Donnell, M.A. and A. Boehle, 2006. Treatment options for BCG failures. World J. Urol., 24: 481487. PMID: 17021823

O'Garra, A., G. Stapleton, V. Dhar, M. Pearce and J. Schumacher et al., 1990. Production of cytokines by mouse B cells: B lymphomas and normal B cells produce interleukin 10. Int. Immunol., 2: 821832. PMID: 1703785

O'Garra, A. and P. Vieira, 2007. T(H)1 cells control themselves by producing interleukin-10. Nat. Rev. Immunol., 7: 425-428. PMID: 17525751

O'Garra, A and K.M. Murphy, 2009. From IL-10 to IL12: how pathogens and their products stimulate APCs to induce $\mathrm{T}(\mathrm{H}) 1$ development. Nat. Immunol., 10: 929-932. PMID: 19692989

Prescott, S., K. James, T.B. Hargreave, G.D. Chisholm and J.F. Smyth, 1992. Intravesical Evans strain BCG therapy: Quantitative immunohistochemical analysis of the immune response within the bladder wall. J. Urol., 147: 1636-1642. PMID: 1593713 
Qin, Z., G. Noffz, M. Mohaupt and T. Blankenstein, 1997. Interleukin-10 prevents dendritic cell accumulation and vaccination with granulocytemacrophage colony-stimulating factor genemodified tumor cells. J. Immunol., 159: 770-776. PMID: 9218594

Ratliff, T., 1991. Bacillus Calmette-Guérin (BCG): Mechanism of action in superficial bladder cancer. Urology, 37: 8-11. PMID: 2021034

Richter, G., S. Kruger-Krasagakes, G. Hein, C. Hüls and E. Schmitt et al., 1993. Interleukin 10 transfected into Chinese hamster ovary cells prevents tumor growth and macrophage infiltration. Cancer Res., 53: 4134-4137. PMID: 8364905

Riemensberger, J., A. Bohle and S. Brandau, 2002. IFN-gamma and IL-12 but not IL-10 are required for local tumour surveillance in a syngeneic model of orthotopic bladder cancer. Clin. Exp. Immunol., 127: 20-26. PMID: 11882028

Riley, J.K., K. Takeda, S. Akira and R.D. Schreiber, 1999. Interleukin-10 receptor signaling through the JAKSTAT pathway. Requirement for two distinct receptor-derived signals for anti-inflammatory action. J. Biol. Chem., 274: 16513-16521. PMID: 10347215

Rowbottom, A.W., M.A. Lepper, R.J. Garland, C.V. Cox and E.G. Corley, 1999. Interleukin-10-induced CD8 cell proliferation. Immunology, 98: 80-89. PMID: 10469237

Saban, M.R., C. Simpson, C. Davis, G. Wallis and N. Knowlton et al., 2007. Discriminators of mouse bladder response to intravesical Bacillus CalmetteGuerin (BCG). BMC Immunol., 8: 6. PMID: 17506885

Saint, F., J.J. Patard, P. Maille, P. Soyeux and A. Hoznek et al., 2001. T helper 1/2 lymphocyte urinary cytokine profiles in responding and nonresponding patients after 1 and 2 courses of bacillus Calmette-Guerin for superficial bladder cancer. J. Urol., 166: 2142-2147. PMID: 11696723

Saint, F., J.J. Patard, P. Maille, P. Soyeux and A. Hoznek et al., 2002. Prognostic value of a T helper 1 urinary cytokine response after intravesical bacillus Calmette-Guerin treatment for superficial bladder cancer. J. Urol., 167: 364-367. PMID: 11743357

Sakaguchi, S., 2004. Naturally arising CD4+ regulatory $\mathrm{T}$ cells for immunologic self-tolerance and negative control of immune responses. Annu. Rev. Immunol., 22: 531-562. PMID: 15032588
Satyam, A., P. Singh, N. Badjatia, A. Seth and A. Sharma, 2011. A disproportion of TH1/TH2 cytokines with predominance of $\mathrm{TH} 2$, in urothelial carcinoma of bladder. Urol. Oncol., 29: 58-65. PMID: 19837616

Segal, B.M., D.D. Glass and E.M. Shevach, 2002. Cutting Edge: IL-10-producing CD4+ T cells mediate tumor rejection. J. Immunol., 168: 1-4. PMID: 11751938

Sheu, B.C., W.C. Chang, C.Y. Cheng, H.H. Lin and D.Y. Chang et al., 2008. Cytokine regulation networks in the cancer microenvironment. Front Biosci., 13: 6255-6268. PMID: 18508658

Siegel, R., D. Naishadham and A. Jemal, 2012. Cancer statistics, 2012. CA. Cancer J. Clin., 62: 10-29. DOI: $10.3322 /$ caac. 20138

Simons, M.P., M.A. O'Donnell and T.S. Griffith, 2008. Role of neutrophils in BCG immunotherapy for bladder cancer. Urol. Oncol., 26: 341-345. PMID: 18593617

Steinbrink, K., M. Wölfl, H. Jonuleit, J. Knop and A.H. Enk, 1997. Induction of tolerance by IL-10-treated dendritic cells. J. Immunol., 159: 4772-4780. PMID: 9366401

Steinbrink, K., E. Graulich, S. Kubsch, J. Knop and A.H. Enk, 2002. CD4(+) and CD8(+) anergic T cells induced by interleukin-10-treated human dendritic cells display antigen-specific suppressor activity. Blood, 99: 2468-2476. PMID: 11895781

Taniguchi, K., S. Koga, M. Nishikido, S. Yamashita and T. Sakuragi et al., 1999. Systemic immune response after intravesical instillation of bacille Calmett-Guérin (BCG) for superficial bladder cancer. Clin. Exp. Immunol., 115: 131-135. PMID: 9933432

Tanikawa, T., C.M. Wilke, I. Kryczek, G.Y. Chen and J. Kao et al., 2012. Interleukin-10 ablation promotes tumor development, growth and metastasis. Cancer Res., 72: 420-429. PMID: 22123924

Tham, S.M., K.H. Ng, S.H. Pook, K. Esuvaranathan and R. Mahendran, 2011. Tumor and microenvironment modification during progression of murine orthotopic bladder cancer. Clin. Dev. Immunol., 2011: 865684. PMID: 22013484

Tilg, H., C. VAn Montfrans, A. Van Den Ende, A. Kaser and S.J. Van Deventer et al., 2002. Treatment of Crohn's disease with recombinant human interleukin 10 induces the proinflammatory cytokine interferon gamma. Gut, 50: 191-195. PMID: 11788558 
Trinchieri, G., 2007. Interleukin-10 production by effector T cells: Th1 cells show self control. J. Exp. Med., 204: 239-243. PMID: 17296790

Wehinger, J., F. Gouilleux, B. Groner, J. Finke and R. Mertelsmann et al., 1996. IL-10 induces DNA binding activity of three STAT proteins (Stat1, Stat3 and Stat5) and their distinct combinatorial assembly in the promoters of selected genes. FEBS Lett., 394: 365-370. PMID: 8830676

Willems, F., A. Marchant, J.P. Delville, C. Gérard and A. Delvaux, et al., 1994. Interleukin-10 inhibits B7 and intercellular adhesion molecule-1 expression on human monocytes. Eur. J. Immunol., 24: 10071009. PMID: 7512027

Williams, S.K., D.M. Hoenig, R. Ghavamian and M. Soloway, 2010. Intravesical therapy for bladder cancer. Expert Opin. Pharmacother., 11: 947-958. PMID: 20205607
Yang, A.S. and E.C. Lattime, 2003. Tumor-induced interleukin 10 suppresses the ability of splenic dendritic cells to stimulate CD4 and CD8 T-cell responses. Cancer Res., 63: 2150-2157. PMID: 12727833

Zeller, J.C., A. Panoskaltsis-Mortari, W.J. Murphy, F.W. Ruscetti and S. Narula et al., 1999. Induction of $\mathrm{CD} 4+\mathrm{T}$ cell alloantigen-specific hyporesponsiveness by IL-10 and TGF-beta. J. Immunol., 163: 3684-3691. PMID: 10490963

Zheng, L.M., D.M. Ojcius, F. Garaud, C. Roth and E. Maxwell et al., 1996. Interleukin-10 inhibits tumor metastasis through an NK cell-dependent mechanism. J. Exp. Med., 184: 579-584. PMID: 8760811 